\title{
Research on Fault Feature Extraction of Rubbing Rotor Based on Vector-Bispectrum Energy
}

\author{
Zhang Chao \\ Department of Mechanical Engineering, North China Electric Power University (071003), Baoding, China
}

\begin{abstract}
(Received 11 December 2014; accepted 10 June 2016)
Signals collected from dual-channel sensors contain abundant fault characteristic information when the rub-impact fault occurs in a rotor system. As a combination of bispectrum and vector-spectrum analisys, vector-bispectrum analysis can achieve an effective elimination of Gaussian noise and accurate analysis on quadratic phase coupling in signals by combining dual-channel information. However, it has been found in the slight rubbing experiment that part of the fault information is lost by simply using the bispectrum or vector-bispectrum method. In order to resolve this problem, a new fault feature extraction approach for the rubbing rotor based on the energy index of vector-bispectrum is proposed and used in the experimental test to obtain typical characteristics of the full annular rub-impact fault. It is shown that this novel method of feature extraction inherits the advantages of vector-spectrum analysis. The features of the rub-impact fault based on the energy index of vector-bispectrum has been extracted successfully, and the result of classification through SVM illustrates that the extracted features are very noticeable, and the proposed method can comprehensively reflect nonlinear information of the rubbing rotor.
\end{abstract}

\section{INTRODUCTION}

In a theoretical sense, the rotor of rotating machinery is a complicated nonlinear system. The occurrence of the rubimpact faults in a rotor system may result in irregular and unstable shafting vibrations containing nonlinear phase coupling, which was harmful to the unit. Typically, conventional methods for fault detection use the power spectrum analysis based on Fourier transformation, which was built on the assumption of stable and Gaussian signals. Spectrum analysis could be used to characterize the Gaussian process, but it was not suitable for processing non-minimum phase systems or non-Gaussian signals due to its incapability of reflecting Gaussian skewness and nonlinear characteristics of signals. ${ }^{1}$ Consequently, developing better ways to analyze the fault of the rubbing rotor has attracted much attention in the field of fault diagnosis of rotating machinery.

High order statistics analysis, in contrast with power spectrum analysis, could suppress Gauss noise completely in theory, and meanwhile effectively reveal the nonlinear information of the signal. As the simplest and lowest-order means in the high order statistics, bispectrum was the most commonly used high-order spectrum, and can be applied to deal with nonlinear phase coupling existing in nonlinear vibration signals. In the current study, bispectrum was generally used in the extraction of fault features in double frequency domain for rotating elements, such as gears and bearings. ${ }^{2-6}$ Shen et al. applied bispectrum to extract the features of the rub-impact fault, and the results indicated the obvious existence of differences in bispectrum between the $X$-direction and the $Y$-direction signals. ${ }^{7}$ However, why only the $Y$-direction signals were not selected for analysis was not explained. Yan et al. adopted the method of diagonal slice of bispectrum to analyze nonlinear coupling of harmonics occurred in steam turbines, but the results showed that the difference between the stable and the unstable vibrations was not notable in the figure of diagonal slice of bispectrum. ${ }^{8}$ Generally, fault diagnostics based on bispectrum used the method of the statistical change detection, which was not ideal to distinguish the fault degree. ${ }^{9}$ Therefore bispectrum coupled with other methods such as HHT or LMD, were suggested, but the complexity of the algorithm would in- creased. ${ }^{10-13}$

In general, the monitoring system of a rotor had a couple of sensors at each measuring point. The aforementioned studies mostly computed bispectrum of signal per channel respectively and selected the most obvious difference as the fault features by intercomparison. In fact, the signals of different directions were not identical due to the anisotropy of rotor systems. Hence, single channel based bispectrum analysis was not enough to comprehensively and exactly reflect the fault features because the insufficient signal combination would lead to the fault information missing. Based on the research of vector spectrum, ${ }^{14}$ a new method of vector-spectrum integrating dualchannel signals was discussed and used to extract the feature of fault signals. ${ }^{15}$ However, applications of this method carried out by the author of this paper for the fault signals of the rubbing rotor showed that the fault features reflected by vectorbispectrum were not obvious enough to identify the rubbing rotor.

In short, a new method of feature extraction based on the energy index of vector-spectrum was proposed for the rubbing rotor in this paper. The experiment results show that the energy index of vector-spectrum obtains bispectral characteristics of the full annular rub-impact fault, and a reasonable explanation is given. Finally, a SVM classifier is developed to validate the effectiveness of this method.

\section{PRINCIPLE OF VECTOR-SPECTRUM}

Assume that $X$ - and $Y$ - are the two orthogonal directions at a measuring point in a rotor system, and $x_{k}, y_{k}$ are the discrete time series of $X$ - and $Y$-direction respectively. $X_{k}$ and $Y_{k}$ are defined as the Fourier transforms of $x_{k}$ and $y_{k}$. Let $R_{x k}, I_{x k}$, $R_{y k}$, I yk as the real and the imaginary parts of $X_{k}$ and $Y_{k}$, respectively. The amplitude and phase of harmonics included in $x_{k}$ and $y_{k}$ are defined by:

$$
\left\{\begin{array}{l}
A_{x k}=\sqrt{R_{x k}^{2}+I_{x k}^{2}} \\
\varphi_{x k}=\arctan \left(I_{x k} / R_{x k}\right) \\
A_{y k}=\sqrt{R_{y k}^{2}+I_{y k}^{2}} \\
\varphi_{y k}=\arctan \left(I_{y k} / R_{y k}\right)
\end{array}\right.
$$


where $A_{x k}, A_{y k}$ are the amplitudes, and $\varphi_{x k}, \varphi_{y k}$ are the phases of $k$-th harmonic in the $X$ - and $Y$-direction, respectively. Note that the value range of $\varphi_{x k}$ or $\varphi_{y k}$ should be $[-\pi,+\pi]$ in Eq. (1), according to the positive and negative of $I_{k}$ or $R_{k}$ instead of limited in a single monotonous zone $(-\pi / 2,+\pi / 2)$ of arctan function.

According to the function:

$$
A_{k}=\sqrt{A_{x k}^{2}+A_{y k}^{2}} .
$$

In the method of harmonic fusion described by Li, et al., ${ }^{14}$ the signals fusing the $X$ - and $Y$-directions are constructed as follows:

$$
\begin{gathered}
X_{k}^{\prime}=A_{k}\left(\cos \varphi_{x k}+j \sin \varphi_{x k}\right) \\
Y_{k}^{\prime}=A_{k}\left(\cos \varphi_{y k}+j \sin \varphi_{y k}\right) ;
\end{gathered}
$$

where $A_{k}$ is the amplitude of harmonic fusion and $X_{k}^{\prime}, Y_{k}^{\prime}$ are the fusion signals in the $X$ - and $Y$-direction, respectively.

New discrete time series $x_{k}^{\prime}$ and $y_{k}^{\prime}$ were computed by the inverse Fourier transforms of $X_{k}^{\prime}$ and $Y_{k}^{\prime}$. The new time series reflected nonlinear characteristic information in the original signals by fusing the dual-channels data. ${ }^{14}$ The third-order cumulant functions of $x_{k}^{\prime}$ and $y_{k}^{\prime}$ were written as follows:

$$
\left\{\begin{array}{l}
C_{3 x}^{\prime}\left(\tau_{1}, \tau_{2}\right)=E\left[x^{\prime}(k) \cdot x^{\prime}\left(k+\tau_{1}\right) \cdot x^{\prime}\left(k+\tau_{2}\right)\right] \\
C_{3 y}^{\prime}\left(\tau_{1}, \tau_{2}\right)=E\left[y^{\prime}(k) \cdot y^{\prime}\left(k+\tau_{1}\right) \cdot y^{\prime}\left(k+\tau_{2}\right)\right]
\end{array}\right.
$$

where $C_{3 x}^{\prime}\left(\tau_{1}, \tau_{2}\right)$ and $C_{3 y}^{\prime}\left(\tau_{1}, \tau_{2}\right)$ are the third-order cumulant functions of $x_{k}^{\prime}$ and $y_{k}^{\prime}$, and $E$ is the mathematical expectation function.

Then, the two-dimensional Fourier transform of $C_{3 x}^{\prime}$ and $C_{3 y}^{\prime}$ can be computed by:

$$
\left\{\begin{array}{l}
B_{x}^{\prime}\left(\omega_{1}, \omega_{2}\right)=\sum_{\tau_{1}} \sum_{\tau_{2}} C_{3 x}^{\prime}\left(\tau_{1}, \tau_{2}\right) e^{-j\left(\omega_{1} \tau_{1}+\omega_{2} \tau_{2}\right)} \\
B_{y}^{\prime}\left(\omega_{1}, \omega_{2}\right)=\sum_{\tau_{1}} \sum_{\tau_{2}} C_{3 y}^{\prime}\left(\tau_{1}, \tau_{2}\right) e^{-j\left(\omega_{1} \tau_{1}+\omega_{2} \tau_{2}\right)}
\end{array} ;\right.
$$

where $B_{x}^{\prime}\left(\omega_{1}, \omega_{2}\right)$ and $B_{y}^{\prime}\left(\omega_{1}, \omega_{2}\right)$ are the vector-bispectrums of the original signals $x_{k}$ and $y_{k}$. They provided an integration of amplitude information from dual channels maintaining phase information of original signals unchanged. Vectorspectrum inherited the advantages of bispectrum, so it could also remove the Gaussian noise from the original signal to identify the components of the quadratic phase coupling, then the nonlinear information in signals would be indicated.

Since vector-bispectrum is a two-dimensional function with extensive computational efforts, the method of diagonal slice of vector-bispectrum was suggested in practical use to speed up the calculation. Considering a simplified case where $\tau_{1}=\tau_{2}$ in Eq. (5), a one-dimensional function as follows can be obtained.

$$
\left\{\begin{array}{l}
C_{3 x}^{\prime}(\tau)=E\left[x^{\prime}(k) \cdot x^{\prime}(k+\tau) \cdot x^{\prime}(k+\tau)\right] \\
C_{3 y}^{\prime}(\tau)=E\left[y^{\prime}(k) \cdot y^{\prime}(k+\tau) \cdot y^{\prime}(k+\tau)\right]
\end{array} ;\right.
$$

where $C_{3 x}^{\prime}(\tau)$ and $C_{3 y}^{\prime}(\tau)$ are the diagonal slices of the thirdorder cumulant functions of $x_{k}^{\prime}$ and $y_{k}^{\prime}$.

The diagonal slice for vector-bispectrum can be obtained by one-dimensional Fourier transform of Eq. (6) as:

$$
\left\{\begin{array}{rl}
B_{x}^{\prime}(\omega) & =\sum_{\tau_{1}} C_{3 x}^{\prime}(\tau) e^{-j \omega \tau} \\
B_{y}^{\prime}(\omega) & =\sum_{\tau_{1}} C_{3 y}^{\prime}(\tau) e^{-j \omega \tau}
\end{array} .\right.
$$

It should be noted that the diagonal slice of bispectrum preserves the information of high order statistic and quadratic phase coupling with low computational complexity, and was frequently used to extract fault features. ${ }^{8,12}$

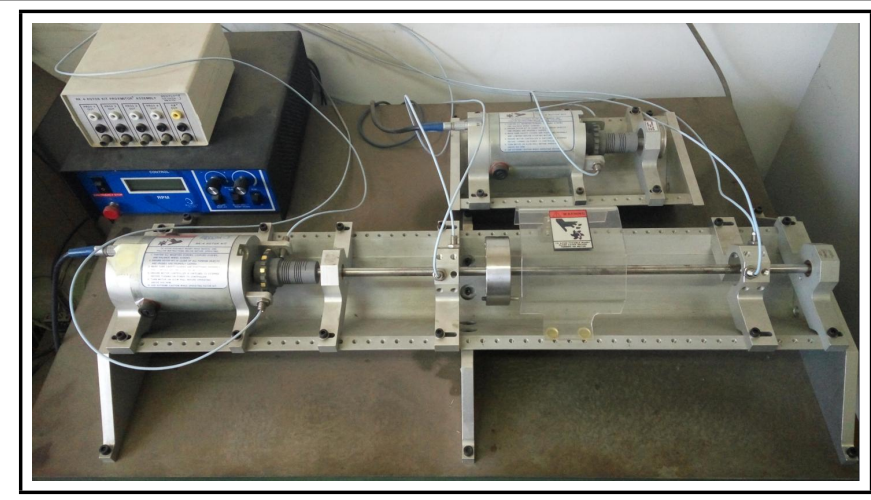

Figure 1. Test apparatus.

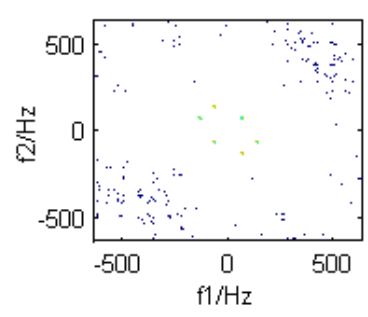

a)

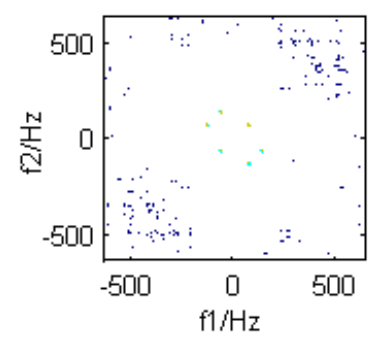

b)
Figure 2. Bispectrum contour of normal rotor.

\section{FEATURE EXTRACTION BASED ON VECTOR-BISPECTRUM ENERGY}

For practical bearings in rotor systems, the stiffness, the damping, and the equivalent of bearings are not the same in different directions. In addition, there are also coupling effects between different coordinates. In other words, the support of a rotor system is anisotropic. When rub-impact faults occur, the characteristic information of the rubbing rotor does not necessarily appear in the diagonal frequencies due to the influence of the anisotropy of bearings. Therefore, it is possible that the diagonal slice cannot exactly extract the fault features of the rub-impact rotor.

In order to verify the statement above, we took an experimental test to simulate the rub-impact fault in the BENTLY NEVADA RK-4 ROTOR KIT, as shown in Fig. 1. Two bearings were installed on a shaft with a single rotor, and an $\mathrm{AC}$ motor was coupled to the shaft to drive the shaft. Two measuring points, each of which was with two eddy current sensors in the orthogonal directions, were set up on both sides of the rotor to collect the radial vibration data. Beside it, we placed another eddy current sensor close to driver to measure the actual rotational speed of the shaft. A plastic rod with adjustable distance was fixed to generate full annular rub to the rotor. The test was conducted repeatedly at different rotational speeds to ensure statistical consistency. We selected the data collected under a constant rotational speed of 4035 RPM (the fundamental frequency $f_{r}=67.25 \mathrm{~Hz}$ ) including 14, 336 samples with a sampling frequency of $1280 \mathrm{~Hz}$. The data length for analysis was 8,192 divided into 16 segments, and the data overlap rate was set to $50 \%$. The bispectrum of selected signals are shown in Figs. 1 and 3.

As seen in Figs. 2 and 3, there are a few differences in bispectrums of the $Y$-direction between the normal and the rubbing rotor in high frequencies. This illustrates that the bispectrum contains some information of rub-impact faults. Also, it 


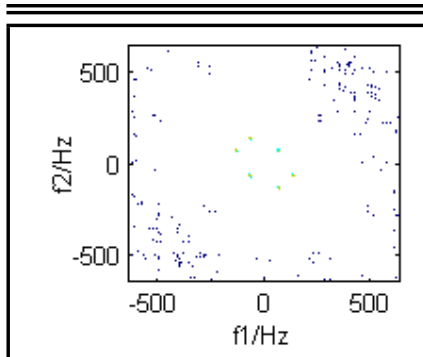

a)

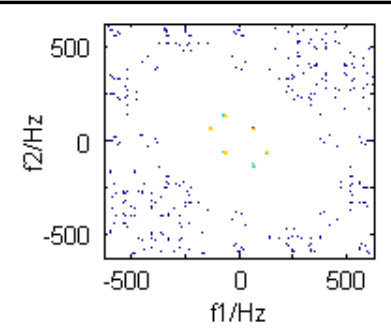

b)

Figure 3. Bispectrum contour of rubbing rotor.

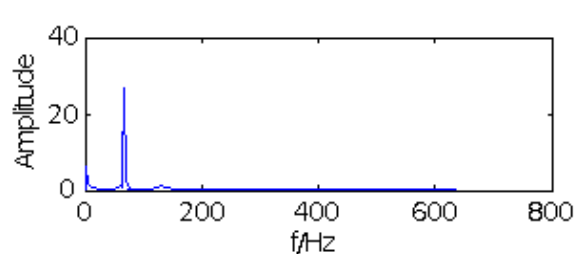

a)

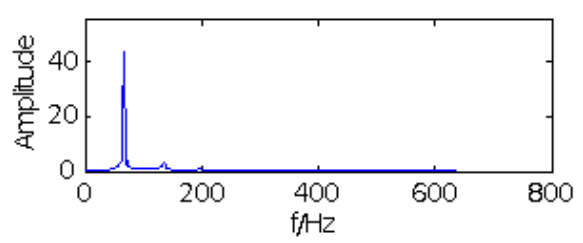

b)

Figure 4. Bispectrum diagonal slice of normal rotor and rubbing rotor.

should be noted that we cannot distinguish the normal rotor from the rubbing rotor via bispectrum of the $X$-direction.

In the above test, we used a plastic rod with an adjustable distance to the rotor in order to simulate the stator and gradually decreased the distance until the plastic rod touched the outer edge of the rotor, and meanwhile maintained a certain force to produce the rub-impact fault. The material of the plastic rod was soft and had a globular head, which would not do any harm to the rotor. In order to simplify the analysis of twodimensional spectrum a diagonal slice of the bispectrum was used to obtain the fault information as shown in Fig. 4. It can be observed in Fig. 4 that the amplitude of bispectrum of the rubbing rotor did not grow much compared with the normal rotor. Therefore, we think that the degree of the rub-impact fault in our experiments would be slight. Note that Fig. 4 has little difference in bispectrum diagonal slices between the normal rotor and the rubbing rotor, which shows that the bispectrum diagonal slice of bispectrum was not capable of reflecting slight rub-impact faults in this case.

Based on the considerations above, we proposed a new method based on vector-bispectrum energy to extract features of the rub-impact faults. According to the method of energy spectrum, ${ }^{16}$ we put forward an extra parameter named index of vector-bisepctrum energy defined by:

$$
G_{z}=\frac{\left|B_{x}^{\prime}\right|^{2}+\left|B_{y}^{\prime}\right|^{2}}{2}
$$

where $G_{z}$ is called the energy index of vector-bisepctrum.

The energy spectrum based on the signal intensity can intuitively reflect the direction of the maximum energy of the rotor in the rotating process with a simple algorithm. This method expands the characteristic frequency information in the diag-

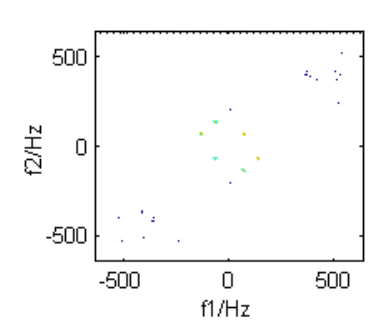

a)

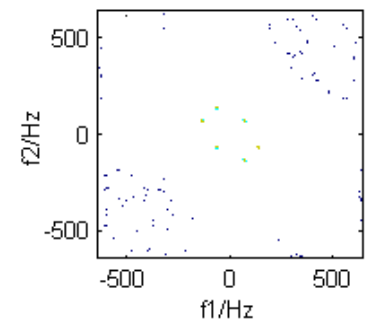

b)
Figure 5. Vector-bispectrum energy contour of normal rotor. a)

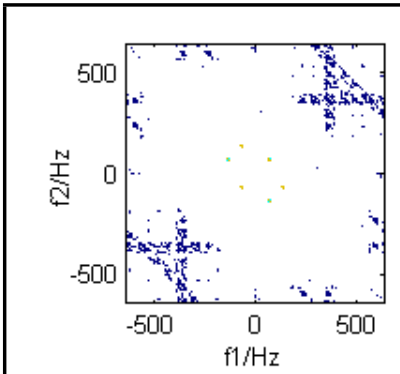

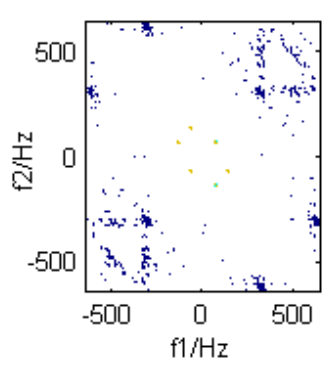

b)
Figure 6. Bispectrum and vector-bispectrum contour of rubbing rotor in $Y$ direction.

onal slice of the vector-bispectrum and is capable of analyzing the characteristic frequency information directly in the frequency plane of the vector- bispectrum, which may dig out the fault information as much as possible.

\section{VECTOR-BISPECTRUM ENERGY INDEX BASED TEST}

According to the construction of the vector-bispectrum, we computed the bispectrum and the vector-bispectrum of the vibration signals at two measuring points respectively, and the results are shown in Figs. 5 and 6.

As indicated in Fig. 5, the scattering pattern of the bispectrum and the vector-bispectrum are similar under the state of the slight full annular rub-impact fault. A further comparison of the vector- bispectrum in the $Y$-direction between Figs. 5 and 6 show that the vector-spectrum implies the existence of faults. It follows that the vector-bispectrum contains more fault information than the bispectrum due to the effective combination of the dual-channel signals, and was able to suppress the Gaussian noise in signals to analyze the characteristics of nonlinear systems superior to the single- channel analysis.

It should be noted that the vibration signals emitted by the rub-impact faults generally contained subharmonic components with appearances of low-frequency couplings. However, we could not find enough subharmonic information as expected in the results produced by the vector-bispectrum and the bispectrum in Figs. 4 and 5, respectively. In order to solve this problem, we used the energy index of vector-bispectrum to reconstitute the contour of vector-bispectrum above. The results are shown in Fig. 7.

In Fig. 7, the subharmonic components of signals are revealed, it illustrates the occurrences of the quadratic phase coupling in the low frequency stage. Comparing Figs. 7a with $7 \mathrm{~b}$, the quadratic phase coupling mainly appears in the low frequency stages during the normal operation of the rotor. In contrast, when the rub-impact fault occurs, the high frequency 


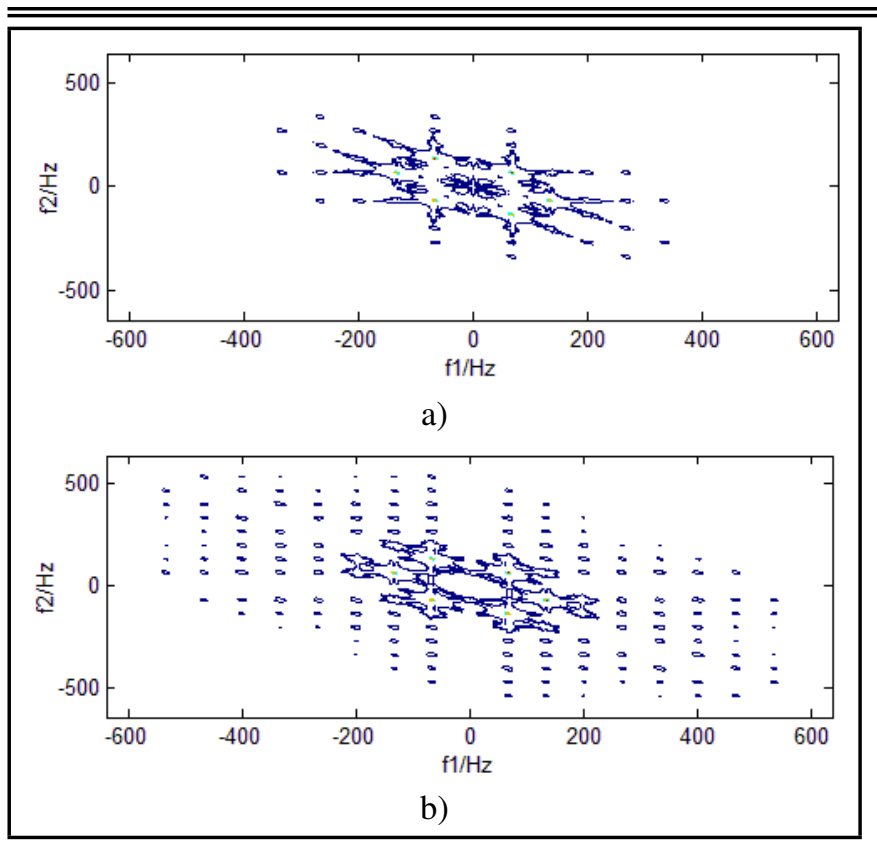

Figure 7. Vector-bispectrum energy contour.

Table 1. Fault recognition ratio with different methods.

\begin{tabular}{|c|c|c|}
\hline \multirow{2}{*}{ Methods } & \multicolumn{2}{|c|}{ Recognition ratio } \\
\cline { 2 - 3 } & Normal & Rubbing \\
\hline Vector-bispectrum & $82 \%$ & $76 \%$ \\
\hline Vector-bispectrum energy index & $96 \%$ & $100 \%$ \\
\hline
\end{tabular}

coupling begins to take place in the vibration signals and will expand to the high frequency stages. However, the components of low frequencies were still in the dominant position. Besides, in Fig. 7b, it can be observed that there are harmonic components that match the fault feature of the rubbing rotor extracted by the conventional method of Fourier transformation.

The diagonal slices for the energy of vector-bispectrum were computed and shown in Fig. 8. Comparing Figs. 8a with 8b, the diagonal slice of the normal rotor is different from the rubbing rotor in frequency distribution. Several harmonic frequencies in the diagonal slice of the rubbing rotor can be observed in Fig. 8b. This indicates that the diagonal slice of the vector-bispectrum energy index contains more fault information than the vector-bispectrum. From the perspective of energy distribution of vector-bispectrum, the energy of the vector-bispectrum mainly centralized in the frequency region below the $2^{\text {nd }}$ harmonic frequency and the largest proportion of the fundamental frequency component indicated the main problem of rotor unbalance. The vector- bispectrum energy of the rubbing rotor is dispersive in the frequency axis with fewer components below the half-frequency. The most concentrated location of energy was in the region between the fundamental frequency and the $4^{\text {th }}$ harmonic frequency, where the quadratic phase coupling came up with the maximum probability.

In order to validate the effectiveness of the fault feature extraction method based on the vector- bispectrum energy index, we applied Support Vector Machine (SVM) as a classifier to distinguish the normal state from the faulty state. In the SVM arithmetic, we used a RBF kernel function, $K\left(x_{i}, x_{j}\right)=$ $\exp \left(-\left|x_{i}-x_{j}\right|^{2} / \sigma^{2}\right), \sigma=0.5$, and selected the amplitude of the bispectrum in $0.5 f_{r}, f_{r}, 2 f_{r}, 3 f_{r}, 4 f_{r}, 5 f_{r}, 6 f_{r}$, and $7 f_{r}$ as the input vectors of SVM. There were 30 samples used to train the SVM classifier and 50 samples for the test. Table 1 illustrates the result of the fault recognition with different methods.

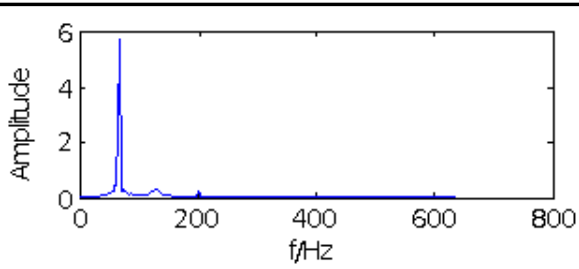

a)

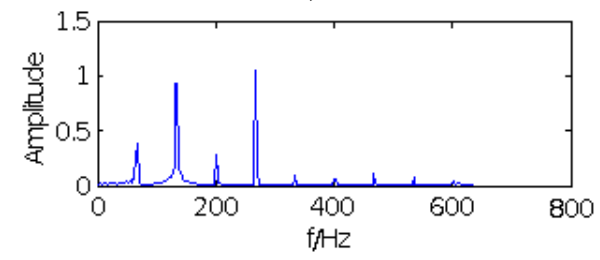

b)

Figure 8. Diagonal slice of Vector-bispectrum energy index.

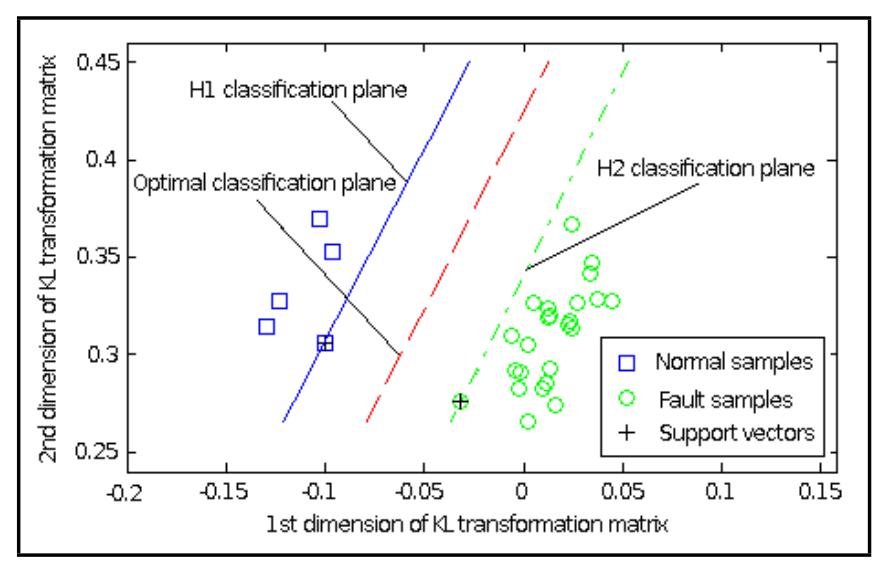

Figure 9. Classification result of SVM after K-L transformation.

Table 1 suggests that the method of vector-bispectrum energy index obtains perfect results of recognition for the rubbing fault, and most normal samples are classified correctly. In order to obtain the visual diagrams of classification, we introduced Karhunen-Loeve transform to compress the eightdimensional input of SVM. Karhunen-Loeve transform is a normalized orthogonal transformation, which can retain main information of the original data. The test data contains 25 samples of the rubbing state and five samples of normal state, and the classification results based on Karhunen-Loeve transform are shown in Fig. 9. As indicated in Fig. 9, it is shown that that all samples are classified correctly.

\section{CONCLUSIONS}

The rubbing rotor is a theoretically nonlinear system. The vibration signals of the rotor are unstable when the quadratic phase coupling happens. To solve this problem, we proposed a novel energy index based on the vector-bispectrum method for feature extraction of rubbing rotors. An experimental test was conducted, where the rub-impact fault features were extracted by the proposed method. The primary conclusions drawn from the study are as follows:

1. The bispectrum can restrain the Gaussian noise and analyze the components of the quadratic phase coupling in the original signals. However, it is found that the bispectrum based feature extraction method does not have any advantages for slight rubbing faults and its diagonal slice may lose fault features. 
2. The vector-bispectrum inherits excellent properties of the bispectrum and meanwhile is more comprehensive than the bispectrum in reflecting the fault information due its information combination of dual-channels. Like the bispectrum, the vector- bispectrum has the probability of losing part of subharmonic components in the slight rubbing fault. The main reason is that the high frequency nonlinear components in vibration signals are in the dominant position, and the subharmonic components do not appear in the resulting figures because they are concealed by high frequency components.

3. The method of vector-bispectrum energy index proposed in this paper is able to distinguish the normal rotor from the rubbing rotor, and the diagonal slice of the vectorbispectrum energy index contains more fault information than the bispectrum to extract fault features from the full annular rub-impact fault rotor. The results of this study indicate that there are components of quadratic phase coupling below the 2nd harmonic frequency during normal operation of rotor. When the rub-impact fault occurs, the components of the quadratic phase coupling will expend to high frequency stages, and the components below the 4 th harmonic frequency are in the dominant position.

\section{ACKNOWLEDGEMENT}

This work is in part supported by National Natural Science Foundation of China (No. 51307058), Natural Science Foundation of Hebei Province, China (No. E2014502052 and No. E2015502013), and Chinese Fundamental Research Funds for the Central Universities (No. 2015ZD27).

\section{REFERENCES}

1 Zheng, H.-B., Chen, X. Z., and Li, Z. Y. Bispectrum based gear fault feature extraction and diagnosis, Journal of Vibration Engineering, 15 (3), 354-358, (2002). http://dx.doi.org/10.3969/j.issn.1004-4523.2002.03.022

2 Shen, G. J., Stephen, M. L., and Xu, Y. C., et al. Theoretical and experimental analysis of bispectrum of vibration signals for fault diagnosis of gears, Mechanical Systems and Signal Processing, 43 (1-2), 76-89, (2014). http://dx.doi.org/10.1016/j.ymssp.2013.08.023

3 Li, X. J, Jiang, L. L., and Yang, D. L., et al. Cluster analysis and fault diagnosis for gear based on bispectrum distribution, Journal of Vibration Engineering, 24 (3), 304-308, (2011). http://dx.doi.org/10.3969/j.issn.10044523.2011.03.014

4 Fidel, E. H. M. and Oscar, C. M. The application of bispectrum on diagnosis of rolling element bearings: A theoretical approach, Mechanical Systems and Signal Processing, 22 (3), 588-596, (2008). http://dx.doi.org/10.1016/j.ymssp.2007.09.003

${ }^{5}$ Li, H., Zheng, H. Q., and Tang, L. W. Gear fault diagnosis based on order bispectrum analysis, Journal of $\mathrm{Ba}$ sic Science and Engineering, 15 (3), 351-356, (2007). http://dx.doi.org/10.3969/j.issn.1005-0930.2007.03.009

6 Zhang, R. G. and Tan, Y. H. Intelligent fault diagnosis of rolling element bearings based on bispectrum principal components analysis, Journal of Vibration Engineering, 27
(5), 763-769, (2014). http://dx.doi.org/10.3969/j.issn.10044523.2014.05.017

7 Wang, L. P., Ye, P. Q., and Wang, J.-S., et al. Bispectrum characteristics of the faults of rubbing rotor system based on experimental study, Journal of Vibration Engineering, 15 (1), 62-67, (2002). http://dx.doi.org/10.3969/j.issn.10044523.2002.01.012

8 Yan, K. G., Liu, Y. B., and Xu, H., et al. Fault feature extraction of large steam turbine based on bispectra analysis of vibration signal, Proceedings of the Chinese Society of Electrical Engineering, 30 (2), 98-103, (2010). http://dx.doi.org/10.13334/j.0258-8013.pcsee.2010.02.011

9 Liu, X. L., Xu, X. L., and Jiang, Z. L., et al. Application of the state deterioration evolution based on bi-spectrum entropy and HMM in wind turbine, Chaos Solitons and Fractals, 89, 160-168, (2016). http://dx.doi.org/10.1016/j.chaos.2015.10.018

10 Lotfi S., Jaouher, B. A., and Farhat, F. Bispectrum based-EMD applied to the non-stationary vibration signals for bearing faults diagnosis, ISA Transactions, 53 (5), 1650-1660, (2014). http://dx.doi.org/10.1109/socpar.2014.7007976

11 Zheng, H., Zhou, L., and Yang, H. Rolling element bearing fault diagnosis based on spectral kurtosis and bi-spectrum, Journal of Beijing University of Aeronautics and Astronautics, 40 (9), 1176-1182, (2014). http://dx.doi.org/10.13700/j.bh.1001-5965.2013.0628

12 Tang, G. J. and Wang, X. L. Fault diagnosis of roller bearings based on local mean decomposition and slice bispectrum, Journal of Vibration and Shock, 32 (24), 83-88, (2013). http://dx.doi.org/10.3969/j.issn.10003835.2013.24.015

13 Yang, J. T. and Zhao, M. Y. Fault diagnosis of traction motor bearings using modified bispectrum and empirical mode decomposition, Proceedings of the Chinese Society of Electrical Engineering, 32 (18), 116-122, (2012). http://dx.doi.org/10.13334/j.0258-8013.pcsee.2012.18.003

14 Li, L. J., Han, J., and Li, P. Y., et al. Vector-bispectrum analysis and its application in machinery fault diagnosis, Journal of Mechanical Engineering, 47 (17), 50-54, (2011). http://dx.doi.org/10.3901/jme.2011.17.050

15 Li, L. J., Han, J., and Li, P.-Y., et al. Intelligent fault diagnosis method based on vector-bispectrum, Journal of Mechanical Engineering, 47 (11), 64-68, (2011). http://dx.doi.org/10.3901/jme.2011.11.064

16 Cheng, J. S., Yang, Y., and Zhang, K., et al. Application of cycle frequency and energy spectrum based on local mean decomposition to gear fault diagnosis, Journal of Vibration Engineering, 24 (1), 78-82, (2011). http://dx.doi.org/10.16385/j.cnki.issn.10044523.2011.01.017 